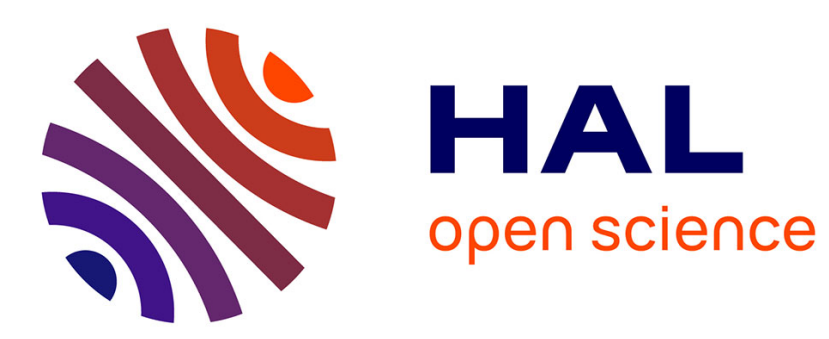

\title{
Market Structure and Insider Trading
}

Wassim Daher, Leonard J Mirman

\section{To cite this version:}

Wassim Daher, Leonard J Mirman. Market Structure and Insider Trading. 2004. halshs-03322686

\section{HAL Id: halshs-03322686 \\ https://shs.hal.science/halshs-03322686}

Submitted on 19 Aug 2021

HAL is a multi-disciplinary open access archive for the deposit and dissemination of scientific research documents, whether they are published or not. The documents may come from teaching and research institutions in France or abroad, or from public or private research centers.
L'archive ouverte pluridisciplinaire HAL, est destinée au dépôt et à la diffusion de documents scientifiques de niveau recherche, publiés ou non, émanant des établissements d'enseignement et de recherche français ou étrangers, des laboratoires publics ou privés. 

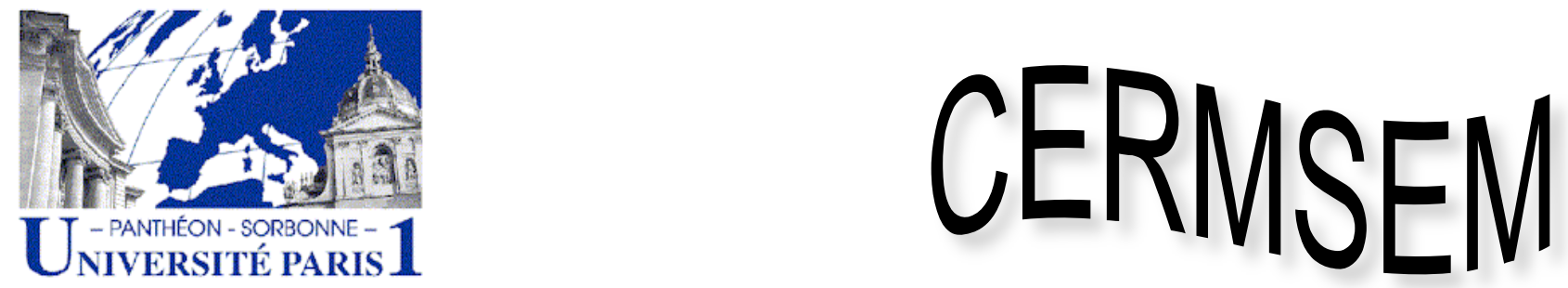

UMR CNRS 8095

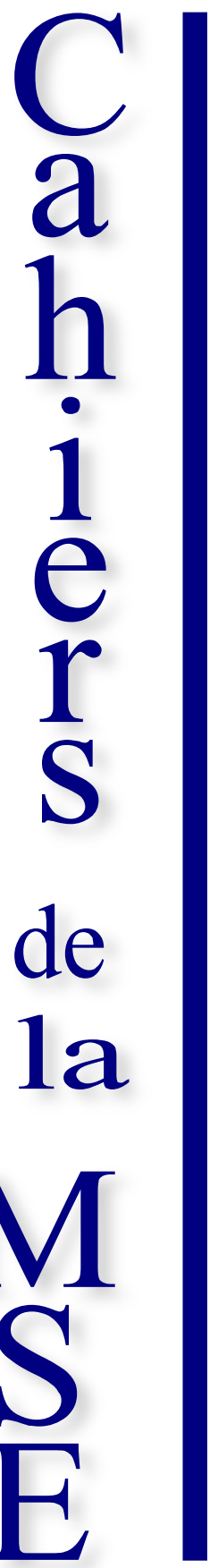

Market Structure and Insider Trading

Wassim DAHER

Leonard J. MIRMAN

2004.25

CENTRE NATIONAL

DE LA RECHERCHE

SCIENTFIQUE

Maison des Sciences Économiques, 106-112 boulevard de L'Hôpital, 75647 Paris Cedex 13 


\title{
MARKET STRUCTURE AND INSIDER TRADING
}

\author{
WASSIM DAHER AND LEONARD J. MIRMAN
}

\begin{abstract}
In this paper we examine the real and financial effects of two insiders trading in a static Jain-Mirman model (Henceforth JM). The first insider is the manager of the firm. The second insider is the owner. First, we study the change of the linear-equilibrium variables, in the presence of two insiders. Specifically, we show that the trading order and the real output of the manager are less in this model than in JM model. Secondly, we show that the stock price reveals more information than in Cournot duopoly and monopoly models studied by Jain-Mirman. Finally, we analyze the comparative statics (insiders' profits) of this model, when the market maker receives one or two signals.
\end{abstract}

Keywords: Insider Trading, Stock prices, Correlated signals, Kyle model

JEL Classification: G14, D82

\section{INTRODUCTION}

Competition has a profound effect on the transmission of information in market economies. This information plays a very important and natural role in influencing the price of securities in a world in which information is decentralized and is not or cannot be made public, except by observing market outputs. In the world of stock markets and firms, there are many different types of competitive interactions that produce information. Insiders are individuals who hold proprietary knowledge about aspects of the firm they are associated with. They may not have managerial responsibilities in the firm, for example, the president or members of the board of directors, with oversight duties but not operational duties. Hence, there are many different types of insiders, each with the objective of maximizing their profits from trading the stock of the firm whose inside information they possess. Hence, one form of competition that influences the stock price, the amount of information disseminated through trading in the stock market as well as the real output decisions of the firm, is the competition among insiders. However this is not the only type of competition that influences the stock price, the transmission of information or the output of the firm. Firms often are in competition with other firms in their market or firms producing substitutes or compliments for their products. This competition has an effect on the real output of the firm. Moreover, it also influences the trading in the stock of the firm as well as the information revealed by the insiders. In this scenario insiders of the firm (those who have information that affects the profitability of the firm) might also include the managers and directors of competing firms. However, as a first approximation,

We thank Joseph Abdou for his helpful comments. 
even without thinking about the effect of insiders on other firms, the competition in the real side of the market affects the financial side of all firms in the market. In sum, there are many types of competition in a complex market with both a real and a financial sides.

Competition among insiders, has an effect on both the financial and the real part of the firm. It is the purpose of this paper to study these real and financial interactions in a model in which insiders trade on their information, while some insiders are involved in the output decisions of the firm and others are not. The effect of this competition in the stock market is the focus of this paper. We present a model that is consistent with the microstructure literature and add competition among informed traders to study the effect of this competition on the equilibrium outcome.

Most of the theoretical literature on insider trading focuses on the financial market, like Kyle [8], Tighe [13], Rochet and Vila [12] and Holden and Subrahmanyam [4]. ${ }^{\text {. Some }}$ recent works like Dow and Rahi [2], Leland [9], Manove [10] and Jain and Mirman [5] and [6], hereafter JM and JMC respectively, incorporate real as well as financial sectors in their models of insider trading. The relationship between the real and financial effects of insider trading has recently been studied by JM and JMC, who focus on the interplay between the information flow from the insider and the real and financial variables of the model, e.g. the stock price and the real output of the firm. This generalization of the Kyle model, allows one to study a rich class of relationships as well as interesting comparative statics.

In a series of papers, Jain and Mirman extend the Kyle model to study, in a more general environment, the effect of information revelation on the trades of an insider, as well as the effect on the real output of the firm. In the first paper, Jain and Mirman (henceforth JMJ) [7], the market maker receives two signals: the total order flow and a signal about the asset's value. Otherwise, the model is the same as the Kyle model. In this paper, it is shown that the amount of information generated by the additional signal is greater than in Kyle and depends on the parameters of the model, e.g., the variances of the respective signals. JM and JMC study a static Kyle-like model in which they assume that the insider is also the manager of the firm. With this market structure, the real and financial effect of insider trading is studied in two cases: monopoly and Cournot duopoly. In both models, they study the unique linear equilibrium of the model, i.e., the market maker sets the stock price as a linear function of the signals he receives. In the monopoly model they examine the effect of the real output produced by the firm, the decision of the insider in the stock market, and the information revealed by the stock price. In the Cournot duopoly, they focus on the linear equilibrium in the presence of two competitive firms, i.e., competition in the real sector. Firm 1 is assumed to be managed by the insider and firm 2 is a standard neoclassical firm with its financial decisions ignored. In both of these models, since the manager makes two decisions, the stock trade and the real output decision, a second order condition in terms of a compensation package for the manager must be imposed in order that the managers objectives are aligned with the objectives of the firm.

\footnotetext{
${ }^{1}$ For excellent surveys, see O'Hara[11].
} 
In the monopoly model, it is shown that, due to this compensation scheme, the insiders trading strategy differs from the insiders trading strategy in Kyle. Moreover, as in JMJ, the market maker sets two (interrelated) coefficients in the price function, one for each signal. They show that the output decision of the firm is influenced by the insiders stock trade. It is also shown that the informational content of the stock trade, as in JMJ, is less than Kyle and is a function of the parameters of the model, the variances of the noise terms as well as the real parameters of the demand function. Finally, they prove that the insider's profits are less when the market maker receives two signals than in a Kyle-like model, i.e., when only one signal (the total order flow) is received.

In the Cournot duopoly model, the effect of the competition between the two firms on the linear equilibrium is examined. In particular, the effect of the different market structures on the stock price, the amount of insider trading, on the real output of the firm, on the amount of information generated, on the profits of the insider and the firm, is studied. They find that the total aggregate output in the Cournot duopoly is greater than the output produced in the monopoly model. In contrast, they show that the trade of the manager is the same as in JMC, in other words, the trade of the insider is not affected by the competition in the real sector. In a sense, this result represents the fact that the same amount of information is generated with two firms in the market as with one firm. Although, the real signal is affected by the change in the output in the market, the informational content of the real signal does not change. Finally, they show that profits are lower than under monopoly. However this is due entirely to the Cournot character of the equilibrium.

The implication in the results of the JMC paper is that competition in the real sector has an effect on the equilibrium of the model similar to the traditional Cournot duopoly literature. However, as noted above, there are many ways to introduce competition when the real and the financial decisions of the firm are modeled together. In this paper, in contrast to the real sector competition, we examine the effect of the competition in the stock sector on the linear equilibrium. In fact, we assume that there is another agent referred to, for convenience, as the owner who is also a speculator in the stock sector and has the same information as the manager. As in the monopoly and Cournot duopoly models, we show that the stock price reveals more information when the market maker receives two signals (the signal from the real market and the total order flow) than when only one signal is received, as Kyle (the total order flow). However, as alluded to in JMC, this signal adds no new information. On the other hand, this type of competition among insiders changes the equilibrium in many ways. First the trading strategies of the two insiders are different from each other. The trading strategy of the owner resembles the one in Kyle, while the trading strategy of the manager resembles the one in JM. Also, the informational structure of the model is different from JMC, since the introduction of an additional trader increases the informational content of the total trades. In some way this result is a combination of JM and Kyle with two traders.

There are other differences between the two models. Competition in the stock sector changes all of the coefficients of the stock price function. Indeed, in the monopoly and 
Cournot duopoly models, the intercept of the price function is zero in both JM and JMC. This is, in fact, a necessary and sufficient condition for the existence of the linear equilibrium in these models. In this model, we find that a necessary and sufficient condition for the existence of a linear equilibrium is that the price function intercept must be equal to the compensation scheme of the manager. This is due to the fact that the compensation of the manager is affected by the existence of a second trader and, thus, changes the ability of the manager to adversely affect the firm. We show that the other coefficients of the price function do not completely represent the information in the signals received by the market maker. In fact, as alluded to in JMJ, the coefficients of the price function are found by regressing the value of the firm on the two signals, and then determining an equilibrium, since the coefficients also have an effect in the determination of the contents of these signals. Hence, the coefficients turn out to be influenced by the information in the signals but not determined by them.

Next, we find that the size of the trade of manager is less than the trade of the insider in both, JM and JMC. We also prove that the stock price reveals more information than in the two models. This is due to the presence of two insiders who conveys more information to the market maker than one insider. Thirdly, we show that manager's profits in our model are less than his profits in the monopoly. This result follows directly from the information received by the market maker remains, and this is true whether the market maker receives one or two signals. Note that the profits of the manager in Cournot duopoly are less than in our model. This is due to the presence of competition in the real sector. Finally, we compare the manager's profits to the owner's profit. We find that the profits of the manager profits and the profits of the owner are not comparable. In other words, the profits of the owner can be greater or less than the managers profits, depending on the value of the firm.

In sum, we show that competition between firms in the real market has a very different affect than competition between insiders in the stock market. Moreover in the latter case, insiders trades are not monolithic. There are differences between insiders trading as well as insiders profits.

\section{THe Model}

Let $(\Omega, \mathcal{F}, \mathbb{P})$ be a probability space, all the random variables are defined with respect to this probability space. We consider a version of the Jain-Mirman (JM) model. There is one real good and one financial asset in the economy. The good in the real sector is produced by a monopolistic firm. The financial asset is the stock of the firm which is publicly traded. We assume that the firm is managed by an agent who decides on the real output of the firm. We add another insider to the JM model, an "owner", 2 without any managerial responsabilities. The owner is assumed to have the same private information about the asset as the manager. The two insiders trade in the stock market based on their

\footnotetext{
${ }^{2}$ What We mean that the "Owner" is another informed agent in the firm. For example a CEO, or a member of the board of directors, who does not have any managerial responsibilities.
} 
inside information. The inverse demand function for the real good is,

$$
q^{\prime}=(a-b y) Z \text {. }
$$

Here $y$ is the real output determined by the manager of the firm, $a$ and $b$ are positive constants and $Z$ is a random variable, normally distributed with mean $\bar{Z}$ (assumed positive) and variance $\sigma_{Z}^{2}$.

The financial asset is traded in a competitive stock market. We assume, as does Kyle (1985), that there are three kinds of agents in the market. First, there are two risk neutral insiders (the manager and the owner of the firm) each of whom knows the realization, $z$, of $Z$. Second, the uninformed liquidity traders (noise traders) who trade randomly for unmodeled reasons. And finally, $K(K \geq 2)$ risk neutral market makers who act like " Bertrand" competitors.

Trading is organized in two steps. First, the market makers determine a pricing function on the basis of their a priori information. This is described by a Lebesgue-measurable function $P: \mathbb{R}^{2} \longrightarrow \mathbb{R}$ (pricing rule). Second, the insiders and noise traders simultaneously, submit their orders, which are based on profit maximization given the public pricing function. In other words, given the pricing function and the realization $z$, each strategic trader chooses a quantity $x_{i}(i=1,2)$, which is a Lebesgue-measurable function $X_{i}$ : $\mathbb{R} \longrightarrow \mathbb{R}$. Let $x_{i}(\omega)=X_{i} \circ Z(\omega), \forall \omega \in \Omega$, the order submitted by insider $i$. We denote by $U$ the total order submitted by noise traders. $U$ is normally distributed with mean 0 and variance $\sigma_{U}^{2}$. The vector $\left(X_{1}, X_{2}\right)$ determines the order flow, $R=X_{1}+X_{2}+U$. Third, the market makers observe the realization of the order flow and a noisy signal $q$ from the real market, but cannot distinguish between the orders of the insiders or the total order of the noise traders. On the basis of this order flow, the signal from the real market and the pricing function, the actual price is determined. Let $p(\omega)=P(q, R)(\omega), \forall \omega \in \Omega$, be this actual price.

2.1. Information Structure and Profit Functions. We assume that $z$ is known by the insiders before making their decisions. The market maker does not observe $z$ but knows its distributions. The market maker also observes both a noisy signal $q$ from the market for the real good and the order flow $R$. Following JM, $q$ is defined as follows,

$$
q=(a-b y)(Z+\varepsilon),
$$

where $\varepsilon$ is normally distributed with mean 0 and variance $\sigma_{\varepsilon}^{2}$. The insiders do not observe $\varepsilon, Z+\varepsilon, U$ and $R$. We assume that $U$ is independent of $\varepsilon$ and $Z$.

Let,

$$
v^{\prime}=(a-b y) y Z,
$$

be the value of the firm, i.e the net profit per share. We denote $\Pi_{1}$ and $\Pi_{2}$ as the trading profits of the manager and the owner, respectively. That is,

$$
\Pi_{1}:=\left(v^{\prime}-A-P\right) X_{1}+A X_{1} \text { and } \Pi_{2}:=\left(v^{\prime}-A-P\right) X_{2},
$$

where $A$ is the compensation the manager receives for his managerial tasks. When the manager receives his compensation, the value of the firm becomes, 


$$
v=v^{\prime}-A
$$

2.2. The Equilibrium Concept. In this section, we introduce the equilibrium notion used in the paper. To begin, we define conditions to be satisfied for a Bayesian-Nash equilibrium for this model. Then we restrict our search to a linear equilibrium. The equilibrium conditions are:

(i) Competition between market makers drives their expected profits to zero.

(ii) The strategy for each informed trader is chosen to maximize expected profits.

Note that in this paper, we assume that condition (i) which is called "Market Efficiency" is fulfilled and derived from unmodeled reasons. A Bayesian-Nash equilibrium of this model is a vector of four functions $\left[y(),. X_{1}(),. X_{2}(),. P(.,).\right]$ such that:

(a) Profit maximization of the manager,

$$
\begin{aligned}
E_{Z}[((a-b y) y z & \left.\left.-P\left(X_{1}(z)+X_{2}(z)+U\right)\right) X_{1}(z)\right] \quad \mathbb{P}_{Z}-a . s . \\
& \geq E\left[\left(\left(a-b y^{\prime}\right) y^{\prime} z-P\left(X_{1}^{\prime}(z)+X_{2}(z)+U\right)\right) X_{1}^{\prime}(z)\right],
\end{aligned}
$$

for any level of output $y^{\prime}$ produced by the firm and any alternative trading strategy $X_{1}^{\prime}$

(b) Profit maximization of the owner,

$$
\begin{aligned}
E_{Z}[((a-b y) y z & \left.\left.-A-P\left(X_{1}(z)+X_{2}(z)+U\right)\right) X_{2}(z)\right] \mathbb{P}_{Z}-a . s . \\
& \geq E\left[\left((a-b y) y z-A-P\left(X_{1}(z)+X_{2}^{\prime}(z)+U\right)\right) X_{2}^{\prime}(z)\right],
\end{aligned}
$$

for any alternative trading strategy $X_{2}^{\prime}$,

(c) Semi-strong market efficiency, the pricing rule $P(.,$.$) satisfies,$

$$
P(q, R)=E[v \mid q, R], \text { almost surely. }
$$

An equilibrium is linear if there exists constants $\mu_{0}, \mu_{1}, \mu_{2}$ such that,

$$
\forall q, r, P(q, r)=\mu_{0}+\mu_{1} q+\mu_{2} r .
$$

Note that conditions (1) and (2) define optimal strategies of the insiders, while condition (3) guarantees the zero expected profit for the market maker.

\section{Optimality Results}

We have a game of incomplete information because the market makers, unlike the insiders do not know the realization of $Z$. Now, we solve the maximization problem of each insider, given the assumption of linear equilibrium. The manager chooses $\left(y, X_{1}\right)$ to maximize expected profit, given $Z=z$. So,

$$
\operatorname{Max}_{\left(y, X_{1}\right)} G_{1}
$$

where $G_{1}=E_{Z}[v-P] X_{1}+A \cdot X_{1}=E_{Z}\left[v^{\prime}-P\right] X_{1}$. This is equivalent to,

$$
\underset{\left(y, x_{1}\right)}{\operatorname{Max}}\left[(a-b y) z-\mu_{0}-\mu_{1}(a-b y) z-\mu_{2}\left(x_{1}+X_{2}(z)\right)\right] x_{1}, \quad \mathbb{P}_{Z}-a . s .
$$


The first order necessary conditions are,

$$
X_{1}(z)=\frac{(a-b y)\left(y-\mu_{1}\right) z-\mu_{0}-\mu_{2} X_{2}(z)}{2 \mu_{2}}, \quad \mathbb{P}_{Z}-\text { a.s. }
$$

and

We obtain

$$
X_{1} Z\left(a-2 b y+\mu_{1} b\right)=0
$$

$$
y=\frac{\left(a+\mu_{1} b\right)}{2 b} .
$$

The owner chooses an order $X_{2}$ to maximize his expected utility, given $Z=z$. So,

$$
\operatorname{Max}_{X_{2}} G_{2}
$$

where $G_{2}=E_{Z}[v-P] X_{2}$. This is equivalent to

$$
\operatorname{Max}_{x_{2}}\left[(a-b y) y z-A-\mu_{0}-\mu_{1}(a-b y) z-\mu_{2}\left(X_{1}(z)+X_{2}\right)\right] x_{2}, \quad \mathbb{P}_{Z}-\text { a.s. }
$$

The first order necessary condition is,

$$
X_{2}(z)=\frac{(a-b y)\left(y-\mu_{1}\right) z-\mu_{0}-A-\mu_{2} X_{1}(z)}{2 \mu_{2}}, \quad \mathbb{P}_{Z}-\text { a.s. }
$$

Solving for $X_{1}$ and $X_{2}$ from (5) and (7), we obtain,

$$
X_{1}(z)=\frac{(a-b y)\left(y-\mu_{1}\right) z-\mu_{0}+A}{3 \mu_{2}}, \quad \mathbb{P}_{Z}-a . s .
$$

and

$$
X_{2}(z)=\frac{(a-b y)\left(y-\mu_{1}\right) z-\mu_{0}-2 A}{3 \mu_{2}}, \quad \mathbb{P}_{Z}-\text { a.s. }
$$

Lemma 1. Let $X=X_{1}+X_{2}, X$ is normally distributed and, therefore, $R$ is normally distributed.

Proof: By adding (8) and (9), we find that $X$ is a linear function of $Z$, which implies that it is normally distributed. Since $X_{1}$ and $X_{2}$ are independent of $U$ (by assumption), $X$ is also independent of $U$ and thus the sum $R$, is normally distributed.

Lemma 2. The random vector $B=(v, q, R)$ is normally distributed and, therefore, $E[v \mid q, R]=\mu_{0}+\mu_{1} q+\mu_{2} R$.

Proof: First, note that $y$ is deterministic, hence, $v$ and $q$ are normally distributed. Second, every linear combination of the elements of $B$, is normally distributed. Thus, $B$ is a normal random vector. Finally, we can apply Theorem 1 in the Appendix to the random vector $B$ and then the result is proved.

Let us now determine the coefficients $\mu_{0}, \mu_{1}$ and $\mu_{2}$.

Lemma 3. Under competition in the stock sector, we have,

(i) $\mu_{0}=\bar{v}-\mu_{1} \bar{q}-\mu_{2} \bar{R}$, 
(ii) $\mu_{1}=\frac{a}{b} k$,

(iii) $\mu_{2}=\frac{a^{2} \sigma_{\varepsilon} \sqrt{(1-k)^{3} k}}{2 b \sigma_{U} \sqrt{3}}=\frac{3 \sqrt{2} a^{2} \sigma_{\varepsilon}^{4} \sigma_{z}}{b \sigma_{u}\left(\sigma_{z}^{2}+6 \sigma_{\varepsilon}^{2}\right)^{2}}$,

where $k=\frac{\sigma_{Z}^{2}}{\left(\sigma_{Z}^{2}+6 \sigma_{\varepsilon}^{2}\right)}$.

Proof: See Appendix.

Note that after calculating the value of $\mu_{0}$ from Lemma (3) and substituting in (8), we get,

$$
X_{1}(z)=\frac{(a-b y)\left(y-\mu_{1}\right)(z-\bar{Z})+3 A}{3 \mu_{2}} \quad \mathbb{P}_{Z}-\text { a.s. }
$$

Let us now write the second order condition for each insider. For the manager, the second order conditions are,

$$
\begin{gathered}
2 b X_{1}(z) z>0, \quad \mathbb{P}_{Z}-\text { a.s. } \\
\mu_{2}>0
\end{gathered}
$$

and the Hessian determinant is positive. For the owner,

$$
\mu_{2}>0 \text {. }
$$

Proposition 1. A linear equilibrium exists if and only if,

$$
\left[b\left(y-\mu_{1}\right)^{2}(z-\bar{Z})+3 A\right] z>0, \quad \mathbb{P}_{Z}-\text { a.s. }
$$

Proof: Substituting the value of $X_{1}$ in equation (11), we obtain the result.

Notice that Proposition 1 does not ensure the satisfaction of the second order condition for all possible values of $Z$. Indeed, since the condition in Proposition 1 is quadratic in $Z$, there exits values of $Z$ such that the second order condition is not satisfied. Now, we present a necessary and sufficient condition (11) for the existence of the linear equilibrium for all $Z$.

Lemma 4. A linear equilibrium exists if and only if,

$$
A=\mu_{0}=\frac{(a-b y)\left(y-\mu_{1}\right)}{3} \bar{Z} .
$$

Proof: We start by proving sufficiency. If $A=\mu_{0}$, then the expression for $X_{1}$ in equation (8) becomes,

$$
X_{1}(z)=\frac{(a-b y)\left(y-\mu_{1}\right) z}{3 \mu_{2}}, \quad \mathbb{P}_{Z}-\text { a.s. }
$$

Thus, the second order condition, given in Proposition 1, is satisfied. Hence, from Proposition 1, there exists a linear equilibrium. Next, we prove the necessary condition. Indeed, since there exists a linear equilibrium, the second order condition of Proposition 1 is satisfied for all $Z$. The second order condition has the following form: $c Z^{2}+d Z>0$. Here $c=b\left(y-\mu_{1}\right)^{2}>0$ and $d=3 A-b\left(y-\mu_{1}\right)^{2} \bar{Z}$. So, we must have $d=0$ to satisfy the second order condition (11), and then the result is proved. 
Remark 3.1. First, note that $A$ is positive. Second, if $Z$ is a centered normal random variable $(\bar{Z}=0)$, there is no need, as in JM, for a compensation scheme to satisfy the second order condition. Indeed, if $\bar{Z}=0$, then $A=0$. Hence, in this case, by Proposition 1 , a linear equilibrium exists and is symmetric, in the sense that the two insiders have the same trading orders. It should be pointed out that the effect of the compensation scheme is to center the manager's (random) profit function around 0, this is also the case of the insider in JM. This has the effect of aligning the objective of the shareholder and the manager. However, there is no need for a compensation scheme for the owner since the owner has no effect on the output decision of the firm. Thus, the random profit function of the owner is centered at the mean $\bar{Z}$, as is the case without production e.g., in Kyle. So when $\bar{Z}=0$, both the profit functions of the manager and the owner are centered around zero, the mean of $Z$, and there is no need for a compensation to the manager.

Lemma 4 provides several interesting and important insights. First, as in Kyle but not $\mathrm{JM}, \mu_{0}$ is different from zero. This is due to the presence of competition in the stock sector, which changes the form of the price function. Second, recall that in Kyle, the price is uninformative and equal to the mean value of the asset if and only if the total order flow is zero. This result is due to the fact that the order flow has a zero mean. In contrast, in our model and like JM, the price is uninformative and equal to the mean value of the asset if the two signals are different from zero and equal to some constants. These constants are calculated in Proposition 2. In other words, as in JM the two signals convey information even when they are zero. This was not noted by JM. In both our model and in JM, the mean of the order flow is not equal to zero.

Proposition 2. The price of the stock is uninformative, that is $p=\bar{v}$, if $Z+\varepsilon=\bar{Z}$ and $R=\frac{A \bar{Z}}{\mu_{2}}$

Proof: Since we deal with linear price, it is straightforward to prove this result.

The next proposition presents the unique equilibrium of the model.

Proposition 3. A linear equilibrium exists. The equilibrium is unique and characterized by the following values of $X_{1}, X_{2}, y$ and $P$.

(i) $y=\frac{\left(a+\mu_{1} b\right)}{2 b}$,

(ii) $X_{1}(z)=\frac{(a-b y)\left(y-\mu_{1}\right) z}{3 \mu_{2}}, \quad \mathbb{P}_{Z}-$ a.s.

(iii) $X_{2}(z)=\frac{(a-b y)\left(y-\mu_{1}\right) z-3 \mu_{0}}{3 \mu_{2}}, \quad \mathbb{P}_{Z}-$ a.s.

(iv) $X=X_{1}+X_{2}=\frac{2(a-b y)\left(y-\mu_{1}\right) z-3 \mu_{0}}{3 \mu_{2}}, \quad \mathbb{P}_{Z}-$ a.s.

(v) $P(q, R)=\mu_{0}+\mu_{1} q+\mu_{2} R$,

(vi) $\mu_{0}=A=\frac{(a-b y)\left(y-\mu_{1}\right)}{3} \bar{Z}$, 
(vii) $\mu_{1}=\frac{a}{b} k$,

(viii) $\mu_{2}=\frac{a^{2} \sigma_{\varepsilon} \sqrt{(1-k)^{3} k}}{2 \sqrt{3} b \sigma_{U}}=\frac{3 \sqrt{2} a^{2} \sigma_{\varepsilon}^{4} \sigma_{z}}{b \sigma_{u}\left(\sigma_{z}^{2}+6 \sigma_{\varepsilon}^{2}\right)^{2}}$,

where $k=\frac{\sigma_{Z}^{2}}{\left(\sigma_{Z}^{2}+6 \sigma_{\varepsilon}^{2}\right)}, \mu_{1} \in\left(0, \frac{a}{b}\right)$ and $\mu_{2}>0$.

\section{Comparative Statics}

In this section, we present several comparisons between the results of our model and the results of JM. Note that these comparisons highlight the effects of a change in the market stucture on the real and financial sectors as well as the decisions of the insiders.

4.1. Equilibrium variables and coefficients. In this paragraph we compare the coefficients and the variables of the unique equilibrium of Proposition 3 to those of JM. Note that the notation "jm" refers to the JM model. We, then have the following results,

Lemma 5. Comparing our result to JM, we have,

(i) $\mu_{0}^{j m}=0<\mu_{0}$,

(ii) $\mu_{1}<\mu_{1}^{j m}$

(iii) $\begin{cases}\mu_{2}>\mu_{2}^{j m}, & \text { when } \sigma_{\varepsilon}^{2} \text { is small relative to } \sigma_{Z}^{2}, \\ \mu_{2}<\mu_{2}^{j m}, & \text { otherwise, }\end{cases}$

(iv) $y<y^{j m}$

(v) $X_{1}<X^{j m}$.

Proof: Recall that $\mu_{0}^{j m}=0$ which is less than $\mu_{0}$ (by Lemma 3 and the fact that $\bar{Z}$ is positive). Comparing $\mu_{1}^{j m}=\frac{a \sigma_{Z}^{2}}{b\left(\sigma_{Z}^{2}+4 \sigma_{\varepsilon}^{2}\right)}$ with $\mu_{1}$ given in Lemma 3 , it follows that $\mu_{1}<\mu_{1}^{j m}$. A similar comparison between $\mu_{2}^{j m}$ and $\mu_{2}$ results in $(i i i)$. Note that from Proposition 3 , it follows that,

$$
y=\frac{\left(a+\mu_{1} b\right)}{2 b} \text { and } y^{j m}=\frac{\left(a+\mu_{1}^{j m} b\right)}{2 b} .
$$

It is clear that both real outputs have the same expressions but by ( $i i)$ of this Lemma we get the result. For $(v)$, substituting for $y, \mu_{1}$ and $\mu_{2}$ from Proposition 3 into the expression for $X_{1}$ yields

$$
X_{1}(z)=\frac{\sqrt{2} \sigma_{U}}{2 \sigma_{Z}} z, \quad \mathbb{P}_{Z}-\text { a.s. }
$$

which is less than the trading strategy of the monopolist in JM, where,

$$
X^{j m}(z)=\frac{\sigma_{U}}{\sigma_{Z}} z, \quad \mathbb{P}_{Z}-\text { a.s. }
$$

Lemma 6. Comparing the Owner's trading strategy and the compensation scheme to JM, we have, 
(i) there exists a $z^{-} \in \mathbb{R}^{-}$such that,

$$
\left\{\begin{array}{lll}
X_{2}(z)>X^{j m}(z), & \text { if } z \in\left(z^{-}, 0\right) & \mathbb{P}_{Z}-\text { a.s. } \\
X_{2}(z)<X^{j m}(z), & \text { otherwise. } & \mathbb{P}_{Z}-\text { a.s. }
\end{array}\right.
$$

(ii) there exists $a z^{+} \in \mathbb{R}^{+}$such that,

$$
\left\{\begin{array}{lll}
X(z)<X^{j m}(z), & \text { if } z \in\left(0, z^{+}\right) & \mathbb{P}_{Z}-\text { a.s. } \\
X(z)>X^{j m}(z), & \text { otherwise. } & \mathbb{P}_{Z}-\text { a.s. }
\end{array}\right.
$$

(iii) $A\left(\mu_{1}\right)<A^{j m}\left(\mu_{1}\right)$

Proof: Substituting for $y, \mu_{1}$ and $\mu_{2}$ from Proposition 3 into the expression for $X_{2}$ yields

$$
X_{2}(z)=\frac{\sqrt{2} \sigma_{U}}{2 \sigma_{Z}}(z-\bar{Z}) \quad \mathbb{P}_{Z}-\text { a.s. }
$$

Comparing the expressions in (15) and (16), the result in (i) holds. By adding (14) and (15), we obtain,

$$
X(z)=\frac{\sqrt{2} \sigma_{U}}{\sigma_{Z}} z-\frac{\sqrt{2} \sigma_{U}}{2 \sigma_{Z}} \bar{Z} \quad \mathbb{P}_{Z}-\text { a.s. }
$$

Comparing equation (17) to equation (15), we get the result. For (iii), it is easy to check that

$$
A\left(\mu_{1}\right)=\frac{\left(a-b \mu_{1}\right)^{2}}{12 b} \bar{Z} \text { and } A^{j m}\left(\mu_{1}\right)=\frac{\left(a-b \mu_{1}\right)^{2}}{8 b} \bar{Z},
$$

which proves the result.

Note that the compensation scheme in both this paper and in JM has two components. The first is the structure of the compensation scheme and the second is the effect of the price coefficient, $\mu_{1}$, of the real signal, on the amount of compensation. In equilibrium the amount of the compensation is fixed. However, this construction is used to make comparisons between the two models.

Lemma 5 and Lemma 6 represent a principal part of the main result of this paper. First, note that the level of trading of the manager in this model is less than in JM. This can be seen from Figure 1, which depicts four trading strategies as a function of the realization of $Z$. These are (1) the trading strategy of the manager in JM, (2) the trading strategy of the manager in this paper, (3) the trading strategy of the owner in this paper and (4) the sum of the trades of the manager and the owner in this paper. In part, this result is due to the presence of the compensation scheme (compare the curves 1 and 2 in Figure 1 , they are linear and cross at zero, which is due to the fact that both are subject to a compensation scheme). And, second by the competition in the stock sector, so that curve 1 has a greater slope than curve 2. This is consistent with the result of Kyle, comparing the model with one trader to the model with two traders, each with the same information (see Tighe 1989).

Recall that in the static Kyle model with multiple insiders, the unique linear equilibrium is symmetric in the sense that each insider has the same level of trading. Since the owner 
in our model does not have any activity in the real sector, we obtain a nonsymmetric equilibrium, even if the two insiders have the same information. The trading curve of the manager goes through the origin, as does the trading curve of the manager in JM. However, the trading curve of the owner is an affine transformation of the trading curve of the manager and, as the insiders in the Kyle model, do not go through the origin. Hence, the trading curves of the owner and the manager in JM, cross at some point $z^{-} \in \mathbb{R}^{-}$ (see Figure 1, for curves 1 and 3). This leads to the conclusion that for long trades, the level of trading of the owner is always less than the level of trading of the manager in JM. In contrast, for short trades, the level of trading of the owner is greater than the level of trading in JM when $Z$ belongs to the interval $\left(z^{-}, 0\right)$ and the level of trading of the owner is less than in JM for $z<z^{-}$.

Second, consider the total level of trading of the manager and the owner, that is $X$. Its curve and the curve of the manager in JM, cross at some point $z^{+} \in \mathbb{R}^{+}$(see Figure 1 for curves 1 and 4). So for short trades, the total trade of the insiders is always greater than in JM. For long trades, the total level of trading of the insiders is less than the level of trading of the manager in JM when $Z$ belongs to the interval $\left(0, z^{+}\right)$and the total level of trading of the insiders is greater than in JM for $z>z^{+}$. Note that this result differs from the one in the Kyle model with multiple traders (see Tighe 1989). The key difference is that in our model, the owner has no activity in the real sector and thus the equilibrium is no longer symmetric. Note, finally, that in the presence of two signals, the standard deviation of the noise signal of the real sector, that is, $\sigma_{\varepsilon}^{2}$, has no effect on the trades of the insiders. This result is similar to the result in JM.

Third, the stock price is affected by the competition in the stock market. Specifically, the response $\mu_{1}$ of the market maker to the signal from the real sector, is lower in this model than in JM. However, the coefficient $\mu_{2}$ of the total order flow signal depends on the variances of the exogenous random variables although more information is revealed in this model than in JM. Indeed, $\mu_{2}$ is lower in this model than in JM when the variance of the noise from the real sector, i.e. $\sigma_{\varepsilon}^{2}$, is large, relative to the variance of the value of the firm $\sigma_{Z}^{2}$ and it is less than in $\mathrm{JM}$ if $\sigma_{\varepsilon}^{2}$ is small, relative to $\sigma_{Z}^{2}$. Note that this result is similar to in JMJ when going from one to two insiders. It should be pointed out that both of these values, $\mu_{1}$ and $\mu_{2}$, depend on the real signal, the value of the firm and the order flow. However, the actual value of $\mu_{1}$ also depends on the output of the firm, i.e., the output of the firm affects the real signal and, thus, becomes a factor in determining $\mu_{1}$. That is, the output of the firm also influnces the value of $\mu_{1}$. Since, the output of the firm is less in our model than in JM, the coefficient $\mu_{1}$ in our model is less than in JM. On the other hand, the structure of the function of the coefficient of the order flow, $\mu_{2}$, is different in the two models (cf equations (34) and (23) in this paper and JM respectively) - This is due to fact that $\mu_{1}, y$, as well as the trading strategy determines the structure of $\mu_{2}$. Moreover, the actual value of $\mu_{2}$ depends on the outputs of the two models, which are also different. Finally, the effects of the changes in the variability of the exogenous variables, $Z, \varepsilon$ and $U$ (that is, $\sigma_{Z}^{2}, \sigma_{\varepsilon}^{2}$ and $\sigma_{U}^{2}$ ) as well as the endogenous financial variables, 
the output and the trading orders, are the same as in JM (see JM, Proposition 5 pp-344 and the discussion that follows).

It is convenient to recall expressions for the signals, in order to study their effects on the compensation received by the manager in our model and in JM. We have

$$
\begin{gathered}
q=(a-b y)(Z+\varepsilon), \\
R=X_{1}+X_{2}+U, \\
A=\frac{\left(a-b \mu_{1}\right)^{2}}{12 b} \bar{Z},
\end{gathered}
$$

and

$$
A^{j m}=\frac{\left(a-b \mu_{1}^{j m}\right)^{2}}{8 b} \bar{Z} .
$$

Even if $\mu_{1}<\mu_{1}^{j m}$, we can not infer that $A<A^{j m}$ or $A>A^{j m}$. Indeed, replacing the expression of $\mu_{1}$, as well as the expression of $\mu_{1}^{j m}$, in the expressions of their respective compensation schemes, we obtain,

$$
A=\frac{3 a^{2} \sigma_{\varepsilon}^{4}}{b\left(\sigma_{z}^{2}+6 \sigma_{\varepsilon}^{2}\right)^{2}} \bar{Z},
$$

and

$$
A^{j m}=\frac{2 a^{2} \sigma_{\varepsilon}^{4}}{b\left(\sigma_{z}^{2}+4 \sigma_{\varepsilon}^{2}\right)^{2}} \bar{Z} .
$$

Notice that a change in $\sigma_{\varepsilon}^{2}$, relative to $\sigma_{Z}^{2}$, the variance of the noise signal of the real sector, leads to a change in the compensation schemes of the managers in both our model and JM. Specifically, if $\sigma_{\varepsilon}^{2}$ is large, relative to $\sigma_{Z}^{2}$, the compensation of the manager in our model is less than in JM. In contrast, if $\sigma_{\varepsilon}^{2}$ is small, relative to $\sigma_{Z}^{2}$, the compensation of the manager in our model is greater than in JM. The intuition for this result is the following. When $\sigma_{\varepsilon}^{2}$ is large, relative to $\sigma_{Z}^{2}$, the variance of the signal $q$ is also large, and, thus, the real signal $q$ is less informative relative to the order flow signal. In fact, in this case, the coefficients of the signal of the real sector, $\mu_{1}$, in both models, are essentially the same. On the other hand, $\mu_{2}$, the total order flow coefficient, is large. Hence, for $\sigma_{\varepsilon}^{2}$ large, relative to $\sigma_{Z}^{2}$, the effect of competition in the stock market leads to a lower compensation. In other words, in this case, there is less incentive for the manager to adversely affect the firm when there is competition from the owner in the stock market. This is due to the fact that more information is revealed through the order flow signal in the presence of the competition in stock market. In the case when $\sigma_{\varepsilon}^{2}$ is small, relative to $\sigma_{Z}^{2}$, the opposite is true. That is, the compensation of the manager in our model is greater than in JM. Indeed, when $\sigma_{\varepsilon}^{2}$ is small, relative to $\sigma_{Z}^{2}$, the market maker receives more information from the signal of the real sector than the stock sector. In other words, when $\sigma_{\varepsilon}^{2}$ decreases, in both models, $\mu_{1}$ increases and $\mu_{2}$ decreases (i.e., competition in the financial sector has a weak effect on the revelation of information to the market maker). Moreover $\mu_{1}^{j m}$ becomes 
greater than $\mu_{1}$. Hence, from the expression of the compensation schemes we can infer that the compensation in JM is less than in this paper.

Remark 4.1. First, if we compare our stock price's coefficients $\mu_{1}$ and $\mu_{2}$ in JMC, we find that $\mu_{1}$ takes the same value in both models. Indeed, compare equation (37) in the appendix to the expression of $\mu_{1}$ in Proposition 2, Section 3 in JMC (after correcting this expression). In these two models, both the structure of the coefficient $\mu_{1}$ as well as the output of the two firms differ. These differences cancel each other and, thus, the two models yield the same $\mu_{1}$. Second, note that the compensation scheme of the manager in our model is always greater than JMC. Indeed, recall the expressions of the compensation scheme in JMC,

$$
A^{c}=\frac{\left(a-b \mu_{1}^{c}\right)^{2}}{18 b} \bar{Z}
$$

where the superscript "c" denotes Cournot. Comparing $A^{c}$ and $A$, since the two models have the same $\mu_{1}$, we infer that the compensation of the manager under the competition in the real sector is always less than in our model.

Next, we analyze the effects of the demand parameters ( $a$ and $b$ ) on the equilibrium stock pricing rule, real output and the trading of each insider.

Proposition 4. The effect of the demand parameters on the equilibrium outcome, is,

(i) The coefficient $\mu_{0}$ changes with demand parameters if and only if $\frac{a^{2}}{b}$ changes.

(ii) The coefficients $\mu_{1}$ and $\mu_{2}$ vary positively with a and negatively with $b$.

(iii) Real output varies positively with a and negatively with $b$.

(iv) Manager and owner's trading are independents from $a$ and $b$.

(v) Stock price changes if and only if $\frac{a^{2}}{b}$ changes.

Proof: $(i),\left(\right.$ ii), $($ iii $)$ follow from the expression of $\mu_{0}, \mu_{1}, \mu_{2}$ and $y$ stated in Proposition 3. The result in (iv) follows from the expressions of $X_{1}$ and $X_{2}$ presented in (14) and (16). Finally, substituting the expressions of $\mu_{0}, \mu_{1}$ and $\mu_{2}$ on the expression of equilibrium stock price and using the fact that $q=Z+\varepsilon$ and $R=X_{1}+X_{2}+U$ we obtain the result in $(v)$.

The general properties of the stock pricing function are the same as in JM.

4.2. Informativeness of stock price. In this section we examine the extent to which the stock price reveals information. This issue was originally studied by Kyle (1985) as well as Rochet and Vila (1994). They found that the stock price reveals exactly half of the inside information, regardless of the parameter values of the model. Moreover, recall that in Kyle's model with more than one informed trader, the amount of information revealed increases in the number of traders, ${ }^{3}$ but is still a constant and is independent of the parameter values of the model. This independence result, is due to the fact that the insider has only one option, i.e., either to buy or sell stock. In particular, the insider(s) in these

\footnotetext{
${ }^{3}$ In fact for two traders the amount of information revealed is exactly $\frac{2}{3}$. See Tighe (1989).
} 


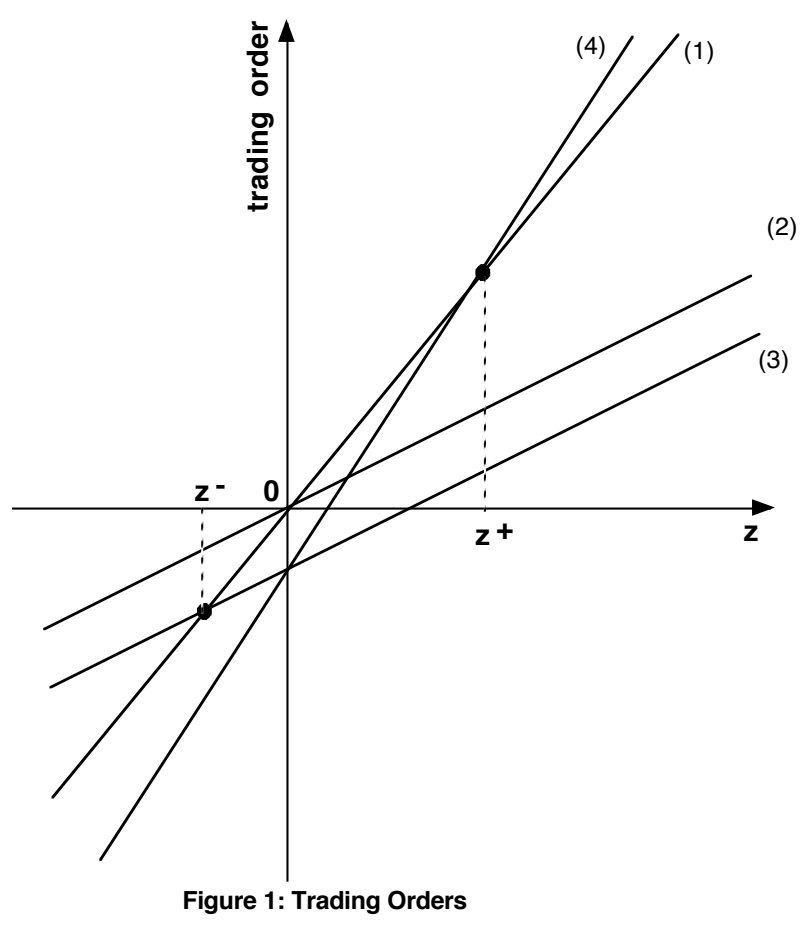

models has no effect on the value of the firm. It is shown in JM that allowing the manager to have an effect on the value of the firm, together with the availability of a second signal to the market maker, changes the result. In particular, in JM, the amount of information released by the insider is greater than half, and, depends on the parameters of the model. In this paper, we show that the stock price reveals more information than in JM, since competition in the stock sector makes the order flow more informative than in JM. On the other hand the informational effect of the real signal is the same as in JM. Recall that the addition of a signal from the real sector, has the effcet of increasing the amount of information recieved by the market maker. However, as discussed below, competition in the stock market has no affect on the informational content of this signal.

Following JM, we adopt the same measure of information i.e., the conditional variance of $Z$, given the information of the market maker. This can be written as, ${ }^{4}$

$$
\begin{aligned}
\operatorname{Var}(Z \mid q, R) & =\sigma_{z}^{2}-\mu_{1} \sigma_{Z q}-\mu_{2} \sigma_{Z R} \\
& =\frac{\left(y-\mu_{1}\right)}{3 y} \sigma_{Z}^{2} .
\end{aligned}
$$

\footnotetext{
${ }^{4}$ See Theorem 1 in the Appendix.
} 
Substituting the expression of $y$ presented in Proposition 3, we get,

$$
\operatorname{Var}(Z \mid q, R)=\frac{\left(a-\mu_{1} b\right)}{3\left(a+\mu_{1} b\right)} \sigma_{Z}^{2}
$$

Note that the coefficient of $\sigma_{Z}^{2}$ is less than half. Hence, as in JM, the observation by the market marker of two signals, (that is $q$, the signal from the real sector and, $R$, the total order flow, from the stock sector) makes the stock price more informative relative to the Kyle (1985) and Rochet and Vila (1994) models. However, we show that there is more information revealed in this paper than in JM.

Proposition 5. The stock price reveals more information in this model than in JM.

Proof: Recall that the conditional variance in JM is,

$$
\frac{\left(a-\mu_{1}^{j m} b\right)}{2\left(a+\mu_{1}^{j m} b\right)} \sigma_{Z}^{2} .
$$

After substituting $\mu_{1}^{j m}$ in expression (19), we get,

$$
\frac{\sigma_{\varepsilon}^{2}}{\sigma_{Z}^{2}+2 \sigma_{\varepsilon}^{2}} \sigma_{Z}^{2}
$$

The conditional variance in our model is given by (18), and after substituting $\mu_{1}$ in expression (18) we get,

$$
\frac{\sigma_{\varepsilon}^{2}}{\sigma_{Z}^{2}+3 \sigma_{\varepsilon}^{2}} \sigma_{Z}^{2}
$$

Comparing expressions (20) and (21), we get the result.

In order to understand the result in more detail, consider the two signals,

$$
q=(a-b y)(Z+\varepsilon),
$$

and

$$
R=X_{1}+X_{2}+U=X+U .
$$

The first thing to note, is that the noise in the order flow signal $R$ is homoskedastic (see Creane 1994), so that the coefficient of $Z$ contained in the total order flow X, does not influence the variance of the noise term $U$. In fact, changing the structure of the model changes the coefficient of $Z$ and thus, the amount of information contained in $R$. In particular, the presence of two inside traders, increases the slope of $X$ over the slope of the inside trades in JM and, thus, $R$ is more informative in this model. While the real signal is heteroskedastic (see Creane 1994) and multiplicative, so that changing the coefficient $(a-b y)$ of $Z+\varepsilon$, does not have any effect on the information contained in this signal. Indeed, the information generated by increasing the slope is offset by the increase in the variance of the signal. Hence, the difference in the information structure of this paper and $\mathrm{JM}$, is only in the order flow signal. Another way of looking at the difference in the two signals is that the effect of increased trading is a factor in the output of the firm, which 
multiplies $Z+\varepsilon$, while the effect on the stock signal is only a multiplier of $Z$. However, the signal from the real sector, that is $q$, cannot be manipulated by the manager after observing the realization, $z$, of $Z$, since the output chosen by the manager is deterministic and, thus, is independent of $Z$. Second, recall that in JM, the manager can manipulate the total order flow, since the total order flow is the sum of his own trade and the noise trades. In contrast, to JM, under competition in the stock sector, the manager is less able to influence the total order flow (recall that the two insiders share the same information).

It should be noted that this discussion shows that the coefficients $\mu_{1}, \mu_{2}$ do not, in general completely reflect the informational content of their respective signals, since, although the real signal in the two models have the same informational content, $\mu_{1}<\mu_{1}^{j m}$. This point was made in JMJ, "The stock pricing coefficients $\mu_{1}$ and $\mu_{2}$ depend in a complicated way on the variances of the two signals as well as their covariances with the value of the firm. Another complicating factor in interpreting $\mu_{1}$ and $\mu_{2}$ is that some of these variances and covariances are also functions of $\mu_{1}$ and $\mu_{2} \ldots "$.

Finally, the intuition for Proposition 5, can also be seen by comparing equations (18) and (19), the conditional variances in our model and in JM, respectively. There are two influences on the conditional variance. The first is the effect of the information contained in the order flow and real signals. This effect shows up in the functional form of the conditional varaince. Hence, for the same $\mu_{1}$, the functional form of JM is greater than in our model. The second is in the values of $\mu_{1}$ and $\mu_{1}^{j m}$. Since, $\mu_{1}<\mu_{1}^{j m}$, the two effects compliment each other.

Remark 4.2. In JMC, even in the presence of the competition in the real sector, it is shown that the stock price reveals the same level of information as in JM. ${ }^{5}$ That is,

$$
\frac{\left(a-\mu_{1}^{c} b\right)}{2\left(a+2 \mu_{1}^{c} b\right)} \sigma_{Z}^{2}
$$

where the superscript $c$ denotes Cournot. This reduces to,

$$
\frac{\sigma_{\varepsilon}^{2}}{\sigma_{Z}^{2}+2 \sigma_{\varepsilon}^{2}} \sigma_{Z}^{2}
$$

This is due to that fact that, the real signal, as discussed above, contains no added information and the order flow in JM and JMC are the same. In this case the value of $\mu_{1}$ in JMC is influenced by the fact that there are two firms controlling the real signal and the coefficient $\mu_{2}$ comes from a signal with the total output which is only being traded. However, this difference is accounted for when taking the conditional variance in both models, which is the same in the two models since the informational content of the signals are the same.

\footnotetext{
${ }^{5}$ Indeed, they find that stock price reveals more information than JM. But, the fault comes from the calculation of $\mu_{1}$ in the Appendix which must be equal to the $\mu_{1}$ of this model. After correcting the expression of $\mu_{1}$ and substitute it in the conditional variance, we obtain the same expression (equation (20)) as in JM.
} 
Remark 4.3. Note that, if the market maker receives only one signal, the total order flow, then the level of information revealed is the same as in Kyle (1985) going from one to two traders. ${ }^{6}$

Proposition 5 highlights the difference between competition in the real sector and competition in the stock sector with respect to the informativeness of the stock price. Indeed, under competition in the stock sector, the stock price reveals more information than the competition in the real sector. This is due to the fact that the change in market structure in JMC has no informational value i.e., more information is not generated in the equilibrium of JMC although there are changes in the equilibrium values of the model. While the changes due to competition in the stock sector affects both the real and the trade signal, although only the change in the stock signal affects the informativeness of the stock price.

Note that the amount of information revealed varies with $\sigma_{Z}^{2}$ and $\sigma_{\varepsilon}^{2}$, a result similar to JM and contrast to Kyle. ${ }^{7}$

\subsection{Insiders Profits.}

4.3.1. Manager's Profit. In this section, we present two results related to the manager's profits. First, we show that the manager's profits in our model are not always less than in JM but they are greater than in JMC. Second, we prove that the profits of the manager in JMC are less than in our paper, which are less than in JM, when the market maker receives only one signal, the total order flow.

In order to prove these results, we calculate the profits of the manager in the presence of two insiders. Substituting for $y, X_{1}, \mu_{0}, \mu_{1}, \mu_{2}$ from Proposition 3, in the manager's profit function, we obtain,

$$
G_{1}(z)=\frac{\left(a-b \mu_{1}\right)^{4} z^{2}}{144 b^{2} \mu_{2}}, \quad \mathbb{P}_{Z}-\text { a.s. }
$$

which reduces to,

$$
G_{1}(z)=\frac{3 \sqrt{2} a^{2}\left(\sigma_{\varepsilon}^{2}\right)^{2} z^{2} \sigma_{U}}{2 b \sigma_{Z}\left(\sigma_{Z}^{2}+6 \sigma_{\varepsilon}^{2}\right)^{2}} . \quad \mathbb{P}_{Z}-\text { a.s. }
$$

Recall the insider's profits in JM and JMC are respectively,

$$
\Pi^{j m}(z)=\frac{\left(a-b \mu_{1}^{j m}\right)^{4} z^{2}}{64 b^{2} \mu_{2}}, \quad \mathbb{P}_{Z}-\text { a.s. }
$$

and

$$
\Pi^{c}(z)=\frac{\left(a-b \mu_{1}\right)^{4} z^{2}}{324 b^{2} \mu_{2}}, \quad \mathbb{P}_{Z}-\text { a.s. }
$$

\footnotetext{
${ }^{6}$ See Tighe (1989). Indeed, it is sufficient to calculate the conditional variance with the variables in Proposition 8 of the next section. It is easy to check that the level of information is $\frac{2}{3}$.

${ }^{7}$ For more details, see JM (2000).
} 
which reduce to,

$$
\Pi^{j m}(z)=\frac{2 a^{2}\left(\sigma_{\varepsilon}^{2}\right)^{2} z^{2} \sigma_{U}}{b \sigma_{Z}\left(\sigma_{Z}^{2}+4 \sigma_{\varepsilon}^{2}\right)^{2}}, \quad \mathbb{P}_{Z}-a . s .
$$

and

$$
\Pi^{c}(z)=\frac{2 a^{2}\left(\sigma_{\varepsilon}^{2}\right)^{2} z^{2} \sigma_{U}}{b \sigma_{Z}\left(\sigma_{Z}^{2}+6 \sigma_{\varepsilon}^{2}\right)^{2}} . \quad \mathbb{P}_{Z}-\text { a.s. }
$$

A comparison between these expressions leads to the following result,

Proposition 6. $\Pi^{c}<G_{1}$

Proposition 7. $\begin{cases}G_{1}>\Pi^{j m} & \text { when } \sigma_{\varepsilon}^{2} \text { is small relative to } \sigma_{Z}^{2}, \\ G_{1}<\Pi^{j m} & \text { otherwise }\end{cases}$

The proof of Propositions 6 and 7 is obvious and therefore is omitted.

Proposition 6 shows that the manager's profits with competition in the real sector are always less than the manager's profit with competition in the stock sector. To see how competition in real sector affects the firm's value, consider equations (22) and (25), representing the respective profits in our model and in JMC. Since, both models have the same $\mu_{1}$, the functional form of the profits in JMC has the same numerator as in our model. However, the total order flow coefficients, that is $\mu_{2}$, in both models, are related in such a way that makes the denominator in the profit function of the manager in JMC is greater than in our model. The reason for this result is that competition in the real sector reduces the firm's value, which is the financial asset of the stock market while competition in the stock sector does not always reduce the value of the firm. Hence, competition in the real sector reduces the value of the firm and, thus, the profits of the manager while competition in the stock sector has an ambiguous effect on the profits of the firm as as on the profits of the manager.

Adding a second informed trader to JM does not always lower the manager's profits relative to JM. ${ }^{8}$ Indeed, Proposition 7 shows that the profits of the manager in this model are greater than in JM when $\sigma_{\varepsilon}^{2}$ is small, relative to $\sigma_{Z}^{2}$ and they are less than JM, when, $\sigma_{\varepsilon}^{2}$ is large, relative to $\sigma_{Z}^{2}$. The intuition for this result is that when $\sigma_{\varepsilon}^{2}$ is large, relative to $\sigma_{Z}^{2}$, the unconditional net profits of firm 1, is less in this model than in JM. In contrast, when $\sigma_{\varepsilon}^{2}$ is small, relative to $\sigma_{Z}^{2}$, the inverse is true, that is the net profits of firm 1 in $\mathrm{JM}$ is less than in this paper. Note that when $\sigma_{\varepsilon}^{2}$ is large, relative to $\sigma_{Z}^{2}$, the total order flow signal $\tilde{r}$ becomes more informative relative to the real signal $\tilde{q}$ and hence this result is similar to the Kyle type models going from one to two insiders (see Tighe 1989).

\footnotetext{
${ }^{8}$ It should be pointed out that in JMJ, going from one to two insiders, the equilibrium is symmetric, i.e., the trading levels of the two insiders are equal and yield equal profits, which are less than the insider's profits in JMJ. This result is due to the absence of the real market, which is studied in this paper but not in JMJ.
} 
In order to make a proper comparison, we now consider as Kyle (1985), the case when the market maker receives only one signal, the order flow. Everything else remains unchanged in our model. The profit function of the manager is

$$
G_{1, k}=E_{Z}[v-P] X_{1}+A X_{1},
$$

where $v=(a-b y) y Z-A$ and the profit function of the owner is,

$$
G_{2, k}=E_{Z}[v-P] X_{2} .
$$

The stock price is set as follows,

$$
P(R)=E[v \mid R]=\mu_{0}+\mu_{1} R .
$$

Solving for this equilibrium we get the following result,

Proposition 8. If the market maker observes only the total order flow, the equilibrium variables become,

(i) $y=\frac{a}{2 b}$,

(ii) $X_{1}(z)=\frac{(a-b y) y z}{3 \mu_{1}}, \quad \mathbb{P}_{Z}$-a.s.

(iii) $X_{2}(z)=\frac{(a-b y) y Z-3 \mu_{0}}{3 \mu_{1}}, \quad \mathbb{P}_{Z}-a . s$.

(iv) $P(R)=\mu_{0}+\mu_{1} R$,

(v) $\mu_{0}=A=\frac{(a-b y) y}{3} \bar{Z}$,

(vi) $\mu_{1}=\frac{\sqrt{2}(a-b y) y \sigma_{Z}}{3 \sigma_{U}}$.

Proof: Following the same method of section 3 for solving the equilibrium variables, and Kyle (1985) to derive the expression of $\mu_{1}$, we get the result.

We can now write the expression of $G_{1, k}$,

$$
G_{1, k}(z)=\frac{a^{2} z^{2} \sigma_{U}}{12 \sqrt{2} b \sigma_{Z}} \quad \mathbb{P}_{Z}-\text { a.s. }
$$

This leads to the following result,

Proposition 9. $G_{1}<G_{1, k}$

Proof: the proof is straightforward.

This result is similar to those obtained in JM and JMC. This is due to the fact that in these models the market maker receives more information ${ }^{9}$ than in Kyle. Hence, he is able to get a better fit of the stock price to the actual value of the firm, thus, the manager's profits from trading, are less than in a Kyle type model. Consequently, the manager's profits when the market maker receives only one signal (the total order flow), are more than the manager's profits when the market maker receives the two signals.

\footnotetext{
${ }^{9}$ The signal from the real sector and the total order flow.
} 
Now, recall the expression of the profit's functions under the same assumptions in JM and JMC respectively. We have,

$$
\Pi_{k}^{j m}(z)=\frac{a^{2} z^{2} \sigma_{U}}{8 b \sigma_{Z}}, \quad P_{Z}-a . s .
$$

and

$$
\Pi_{k}^{c}(z)=\frac{a^{2} z^{2} \sigma_{U}}{18 b \sigma_{Z}} . \quad \mathbb{P}_{Z}-\text { a.s. }
$$

Comparing these expressions we obtain,

Proposition 10. $\Pi_{k}^{c}<G_{1, k}<\Pi_{k}^{j m}$

This is due to the fact that, although, in these models the market maker receives more information than in Kyle, the information contained in the real signal is the same for all three models. Hence, there is no change in the relative amount of information between the three models and, therefore, the manager's profits in this paper are less than in JM when the market maker receives one signal. In other words, competition in the stock sector reduces the profits of the manager by increaing the amount of information. Second, as in Proposition 6, we find that the manager's profits are greater than in JMC. So, even though the market maker receives less information (one signal), the manager's profit in JMC are still less than in this model. The reason is that competition in the real sector influences the value of the firm, which is the financial asset. Hence, competition in the real sector has a stronger effect on the manager's profits than competition in the stock sector. Third, the real signal has an ambiguous effect on the manager's profits. Indeed, in the absence of this signal, adding competition in the stock sector to JM has the same effect on the manager's profits when going from one to two insiders in Kyle type models (see Tighe 1989). Finally, note that the effect of the variability in $Z, \varepsilon$ and $U$ on the manager's profits is the same as in JM.

4.3.2. Owner's Profit. We now show below two properties of the owner's profits. First, as with the manager's profits, we prove that the owner's profits when the market maker receives only one signal (the total order flow), are more than the owner's profits when the market receives the two signals. Second, we compare the manager's profits of this paper to the owner's profits. In order to prove these results, substituting for $y, X_{2}, \mu_{0}, \mu_{1}$ and $\mu_{2}$ from Proposition 3, in the owner's profit function, we get

$$
G_{2}(z)=\frac{3 \sqrt{2} a^{2}\left(\sigma_{\varepsilon}^{2}\right)^{2}(z-\bar{Z})^{2} \sigma_{U}}{2 b \sigma_{Z}\left(\sigma_{Z}^{2}+6 \sigma_{\varepsilon}^{2}\right)^{2}} . \quad \mathbb{P}_{Z}-\text { a.s. }
$$

Let $G_{2, k}$ denotes the owner's profit function when the market maker observes only the total order flow. From Proposition 3, we find that,

$$
G_{2, k}(z)=\frac{a^{2}(z-\bar{Z})^{2} \sigma_{U}}{12 \sqrt{2} b \sigma_{Z}} . \quad \mathbb{P}_{Z}-\text { a.s. }
$$


Comparing the last two expressions, we infer that the owner's profits in this model are less than the owner's profits when the market maker observes only the total order flow. This can be stated as follows,

Proposition 11. $G_{2}<G_{2, k}$

It is easy to interpret this result. As in the previous section, when the market maker receives two signals, he is more able to influence the owner's profits than when he observes one signal, since there is more information generated with two signals. Thus, the owner's profits when the market maker observes two signals are less than his profits when the market maker observes one signal.

Next, we compare the manager's profits to the profits of the owner. First, note that the expected profit function of the manager is quadratic on $z$, the realization of $Z$ and is centered at zero. In contrast, the expected profit function of the owner is quadratic in $z$ but centered at $\bar{Z}$. This can be seen from Figure 2, which depicts the two expected profits as functions of the realization of $Z$. These are (1) the expected profits of the manager and (2) the expected profits of the owner. Hence, the curves representing the expected profits of the two insiders, cross at some point $z_{0} \in \mathbb{R}^{+}$(see Figure 2 for curves 1 and 2 ). This leads to the conclusion that the expected profits of the manager are greater than the expected profits of the owner, for all $z>z_{0}$. And, the expected profits of the owner are greater than the expected profits of the manager for $z<z_{0}$. Second, note that the expected profit functions of the manager in JM as in our model, are both centered at zero. In contrast, the expected profit function of the owner is centered at $\bar{Z}$, a result similar to the one in Kyle (1985). The reason for this result, is that the compensation scheme received by the manager eliminates the presence of $\bar{Z}$ in the manager's expected profit. This is not the case for the owner's expected profit since he has only a financial activities as in Kyle (1985).

\section{APPENDIX}

We start this appendix by recalling the theorem that we use to prove lemma 3 . Then we prove this lemma.

Theorem 1. If the $p \times 1$ vector $Y$ is normally distributed with mean $U$ and covariance $V$ and if the vector $Y$ is partitioned into two subvectors such that $Y=\left(\begin{array}{c}Y_{1} \\ Y_{2}\end{array}\right)$ and if $Y^{*}=\left(\begin{array}{c}Y_{1} \\ Y_{2}^{*}\end{array}\right)$

$$
U=\left(\begin{array}{c}
U_{1} \\
U_{2}
\end{array}\right) \text { and } V=\left(\begin{array}{ll}
V_{11} & V_{12} \\
V_{21} & V_{22}
\end{array}\right)
$$

are the corresponding partitions of $Y^{*}, U$ and $V$, then the conditional distribution of the $m \times 1(m<p)$ vector $Y_{1}$ given the vector $Y_{2}=Y_{2}^{*}$ is the multivariate normal distribution with mean $U_{1}+V_{12} V_{22}^{-1}\left(Y_{2}^{*}-U_{2}\right)$ and covariance matrix $V_{11}-V_{12} V_{22}^{-1} V_{21}$. 


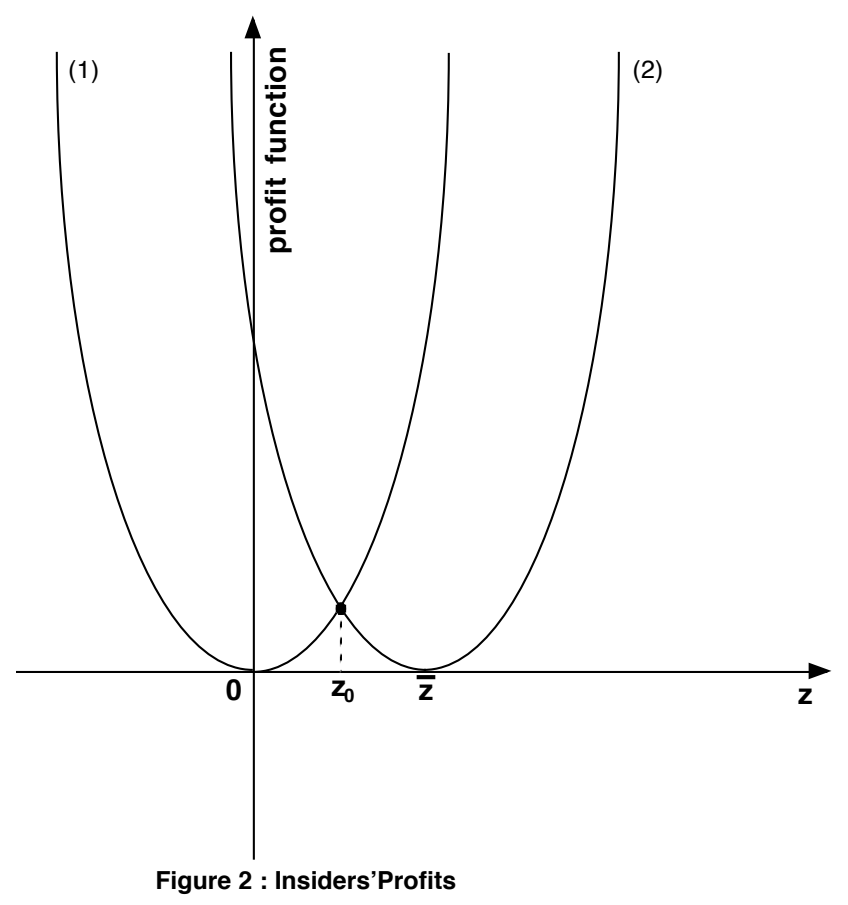

Proof: see Graybill [3], Theorem 3.10 pp 63.

Proof of lemma 3:

We apply theorem 1 to the normal random vector $B=(v, q, R)$. First, in this case we have $p=3$ and $m=1$. Second by identification, we have $Y_{1}=v$ and $Y_{2}=\left(\begin{array}{c}q \\ R\end{array}\right) \cdot U_{1}=\bar{v}$, $U_{2}=\left(\begin{array}{c}\bar{q} \\ \bar{R}\end{array}\right)$ and

$$
V=\left(\begin{array}{ccc}
\sigma_{v}^{2} & \sigma_{v q} & \sigma_{v R} \\
\sigma_{v q} & \sigma_{q}^{2} & \sigma_{q R} \\
\sigma_{v R} & \sigma_{q R} & \sigma_{R}^{2}
\end{array}\right)=\left(\begin{array}{cc}
V_{11} & V_{12} \\
V_{21} & V_{22}
\end{array}\right)
$$

Where $V_{11}=\sigma_{v}^{2}, V_{12}=\left(\sigma_{v q}, \sigma_{v R}\right), V_{21}=\left(\begin{array}{c}\sigma_{v q} \\ \sigma_{v R}\end{array}\right)$ and $V_{22}=\left(\begin{array}{cc}\sigma_{q}^{2} & \sigma_{q R} \\ \sigma_{q R} & \sigma_{R}^{2}\end{array}\right)$

Note that

$$
V_{22}^{-1}=\frac{1}{D}\left(\begin{array}{cc}
\sigma_{R}^{2} & -\sigma_{q R} \\
-\sigma_{q R} & \sigma_{q}^{2}
\end{array}\right)
$$

where $D$ is the determinant of $V_{22}$, that is $D=\sigma_{q}^{2} \sigma_{R}^{2}-\left(\sigma_{q R}\right)^{2}$. 
So we obtain,

$$
\begin{gathered}
\mu_{0}=\bar{v}-\mu_{1} \bar{q}-\mu_{2} \bar{R} \\
\mu_{1}=\frac{\sigma_{v q} \sigma_{R}^{2}-\sigma_{v R} \sigma_{q R}}{D} \\
\mu_{2}=\frac{\sigma_{v R} \sigma_{q}^{2}-\sigma_{v q} \sigma_{q R}}{D}
\end{gathered}
$$

Substituting for the variances and covariances in (31) and (32), we get

$$
\begin{gathered}
\mu_{1}=\frac{(a-b y)^{2} y \sigma_{Z}^{2} \sigma_{U}^{2}}{D} \\
\mu_{2}=\frac{2(a-b y)^{4} y\left(y-\mu_{1}\right) \sigma_{Z}^{2} \sigma_{\varepsilon}^{2}}{3 \mu_{2} D}
\end{gathered}
$$

Computing (33) and (34), we obtain

$$
3 \mu_{2}^{2}=\frac{2(a-b y)^{2} \mu_{1}\left(y-\mu_{1}\right) \sigma_{\varepsilon}^{2}}{\sigma_{U}^{2}}
$$

Calculating for the expression of $D$, we get

$$
D=\frac{4(a-b y)^{4}\left(y-\mu_{1}\right)^{2} \sigma_{\varepsilon}^{2} \sigma_{Z}^{2}}{9 \mu_{2}^{2}}+(a-b y)^{2} \sigma_{U}^{2}\left(\sigma_{\varepsilon}^{2}+\sigma_{Z}^{2}\right)
$$

Substituting the above expression of $D$ in (34), we find

$$
3 \mu_{2}^{2}=\frac{2(a-b y)^{2}\left(y-\mu_{1}\right)\left(y+2 \mu_{1}\right) \sigma_{\varepsilon}^{2} \sigma_{Z}^{2}}{3 \sigma_{U}^{2}\left(\sigma_{\varepsilon}^{2}+\sigma_{Z}^{2}\right)}
$$

Solving (35) and (36), we get

$$
3 \mu_{1}\left(\sigma_{\varepsilon}^{2}+\sigma_{Z}^{2}\right)=\sigma_{Z}^{2}\left(y+2 \mu_{1}\right)
$$

Substituting for $y$ to solve $\mu_{1}$, we obtain

$$
\mu_{1}=\frac{a \sigma_{Z}^{2}}{b\left(\sigma_{Z}^{2}+6 \sigma_{\varepsilon}^{2}\right)}
$$

To solve for $\mu_{2}$, we substitute the above value of $\mu_{1}$ in (35) and taking the positive root, we get

$$
\mu_{2}=\frac{a^{2} \sigma_{\varepsilon} \sqrt{(1-k)^{3} k}}{\sqrt{12} b \sigma_{U}}
$$

where $k=\frac{\sigma_{Z}^{2}}{\sigma_{Z}^{2}+6 \sigma_{\varepsilon}^{2}}$. 


\section{REFERENCES}

[1] A.Creane, Experimentation with Heteroskedastic Noise, Economic Theory, 4, (1994), 275-286.

[2] J. Dow and R.Rahi, Informed Trading, Investment and Welfare, Journal of Business, 76, (2003), 439-454.

[3] F.Graybill An Introduction to Linear Statistical Models: Volume I, McGraw Hill. 1961.

[4] C. Holden and A. Subrahmanyam, Long-Lived Private Information and Imperfect Competition, J.Finance. 47, (1992), 247-270.

[5] N.Jain and L.J Mirman, Real and Financial Effects of Insider Trading with Correlated Signals, Economic Theory. 16, (2000), 333-353.

[6] N.Jain and L.J Mirman, Effects of Insider Trading under Different Market Stuctures, The Quarterly Review of Economics and Finance. 42, (2002), 19-39.

[7] N.Jain and L.J Mirman, Insider Trading with Correlated Signals,Economics Letters. 65, (1999), 105-113.

[8] A. Kyle, Continuous Auctions and Insider Trading, Econometrica. 53, (1985), 1315-1335.

[9] H.E Leland, Insider Trading: Should it be Prohibited, Journal of Political Economy, 100, (1992), 859-887.

[10] M.Manove, The Harm from Insider Trading and Informed Speculation, Quarterly Journal of Economics, 104,(1989), 823-845.

[11] M. O'Hara, "Market Microstructure Theory", Basil Blackwell, Cambridge, Mass. 1995.

[12] J-C. Rochet and J-L.Vila, Insider Trading without Normality,Rev of Econ.Stud, 61,(1994), 131-152.

[13] C. E. Tighe, "Three Essays on Insider Trading" unpublished Ph.D dissertation, University of Illinois at Champaign-Urbana (1989),

Cermsem, Université Paris I, 106-112 boulevard de l'Hôpital, 75647 Paris Cedex 13, FRANCE; DAHER@UNIV-PARIS1.FR.

Departement of Economics, University of Virginia, Charlottesville, VA 22903, USA; LM8H@CMS.MAIL.VIRGINIA.EDU. 